\title{
Automated recognition and sorting of recycled textiles for sustainable fashion
}

\author{
Zlatin Zlatev, Julieta Ilieva \\ Trakia University, Faculty of Technics and Technologies, 8602 Yambol, Bulgaria \\ ${ }^{*}$ Corresponding author E-mail address: zlatin.zlatev@trakia-uni.bg
}

\section{INFO}

CDAPT, ISSN 2701-939X

Peer reviewed article

2021, Vol. 2, No. 2, pp. 151-161

DOI 10.25367/cdatp.2021.2.p151-161

Received: 09 November 2021

Accepted: 03 December 2021

Available online: 09 December 2021

\section{Keywords}

spectral analysis,

textile fibers,

classification,

PCA,

discriminant analysis

\begin{abstract}
The application of the principles of sustainable fashion is one of the solutions to reduce the amount of waste from textile production and the use of such fabrics. Spectrophotometric methods have effective application in this subject area. In the present work, an analysis of known methods and approaches was applied so far using the techniques of spectral analysis. The proposed methods and procedures lead to improvement and facilitation of the process of classification of textile fibers in sorting and recycling of textile fabrics, in order to implement them in automated systems. The proposed analysis tools do not require high-cost equipment and complex calculation procedures. They can be implemented in portable devices and microprocessor-based recognition systems. It has been found that two principal components and two latent variables are sufficient to describe the variance in the data. This significantly reduces the amount of data used to analyze textile fibers by their spectral characteristics. It has been shown that the accuracy of textile fiber classification does not depend on the type of separation function of the classifier used. This accuracy depends on the spectral characteristics used, the method for reducing the volume of data, and the type of classifier. The obtained results can be used in the development of recognition systems for sorting textile fabrics depending on the composition of their fibers. In this way, the principles of sustainable fashion will be effectively applied. Also, the proposed methods and tools can be used in the training of future specialists in the subject area.
\end{abstract}

(C) 2021 The authors. Published by CDAPT.

This is an open access article under the CC BY-NC-ND license https://creativecommons.org/licenses/ peer-review under responsibility of the scientific committee of the CDAPT.

(C) 2021 CDAPT. All rights reserved. 


\section{Introduction}

Retail and fast fashion have been growing in recent years. This in turn leads to a constant increase in the amount of waste from textile production. One solution to this problem is to use the principles of sustainable fashion [1]. These principles are used at all stages of the product lifecycle - from the delivery of materials and the production of textiles, to reaching the end user, and recycling and reuse of textiles.

The active recycling and reuse of textile and fashion waste allows both designers and consumers to use waste as a resource.

The principle of reuse of textile materials is presented in schematic form in Figure 1. The produced textiles are used in design and fashion. Once disposed of by consumers, textile waste is identified by an appropriate automated system and sorted. Sorted textile materials are returned for recycling in textile production. The cycle repeats.

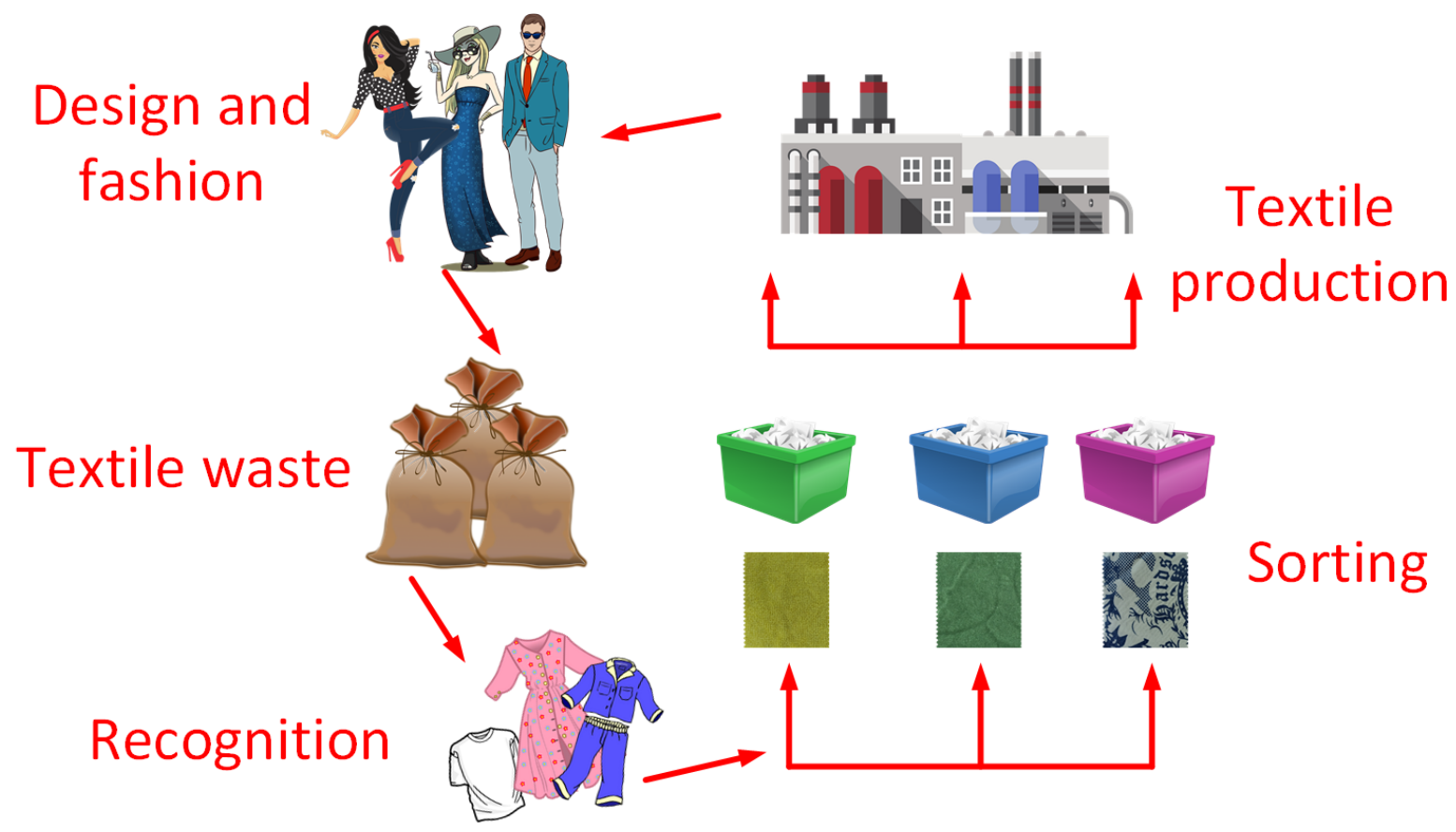

Fig. 1 Cycle for reuse of textile materials

The identification of the types of fibers used in textile fabrics is important when sorting them for recycling.

The fibers used in textile production can be both from natural sources and artificially created, as well as a combination of them. It is possible to sort the textile materials manually, depending on the fibers used. In this case, label data are used. In some cases, the labels may be missing or may not contain information on the textile fibers used [2].

Classical laboratory methods for determining what fibers are used in textile fabrics include dissolution, morphological differences, differential colorimetry, thermogravimetry, gas chromatography [3,4]. Laboratory methods offer sufficient accuracy, but have the main disadvantage that they are time consuming, and sample preparation is required. They are not suitable for use in automated systems for sorting and recycling textile products.

At the current level of development of science and technology, various automated systems for sorting fabrics depending on the fibers used in them are proposed. Optical methods such as microscopic ones, image recognition in digital images, spectral, multispectral and hyperspectral methods are more often used for recognition [5]. The use of methods with digital images has the main disadvantage that the accuracy of textile fiber recognition is significantly influenced by the fabric dyes used. In spectral 
methods, however, the staining of textile fabrics does not significantly affect the recognition accuracy. In some cases, densely applied colorant, as well as darker colors, to some extent affect this recognition [6].

It is necessary to make a more in-depth analysis of the known methods and approaches applied so far using the techniques of spectral analysis, which will lead to improvement and facilitation of the process of classification of textile fibers in sorting and recycling of textile fabrics, in order to implement in automated systems.

\section{Material and methods}

Textile fabrics containing cotton, polyester, elastane, polyamide and various combinations thereof are used. Especially knitted fabrics are used as well as various elastic components which were taken from used clothes. Samples with dimensions $50 \mathrm{~mm} \times 50 \mathrm{~mm}$ were prepared. Additional analyses of surface structure, properties and refinement are included. The materials were used in the period in which they were collected. The captured images and the obtained spectral characteristics are only from one side of the fabric. Figure 2 shows a general view of samples of the fabrics used.

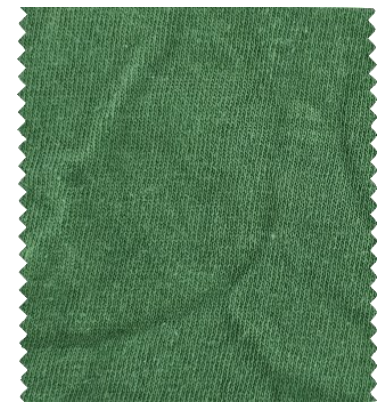

$100 \%$ Cotton

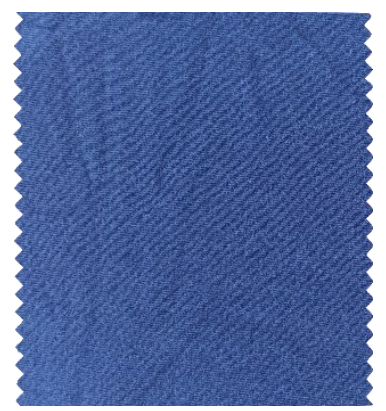

95\% Cotton

$5 \%$ Elastane

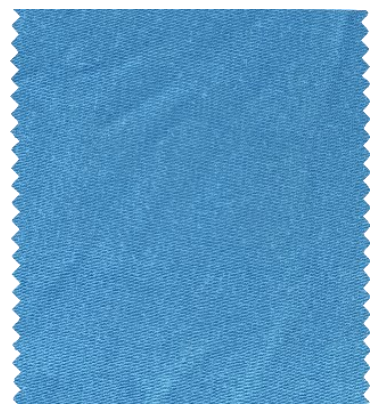

$100 \%$ Cotton

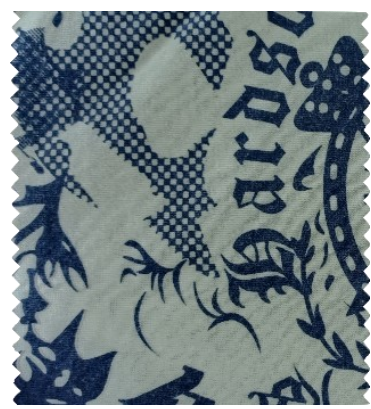

$85 \%$ Cotton

15\% Polyester

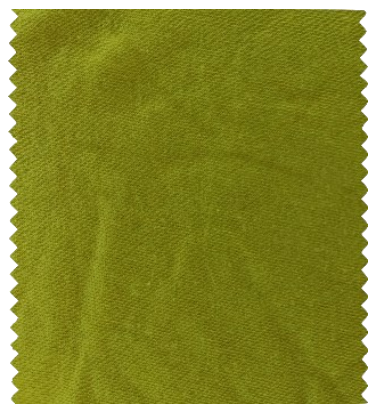

$100 \%$ Cotton

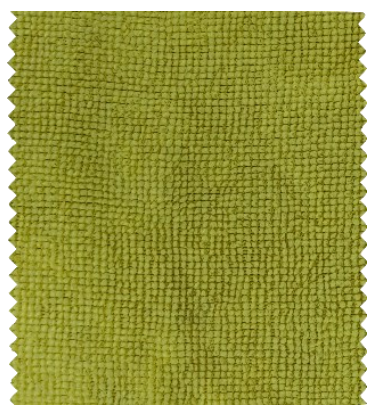

$85 \%$ Polyester

$15 \%$ Polyamide

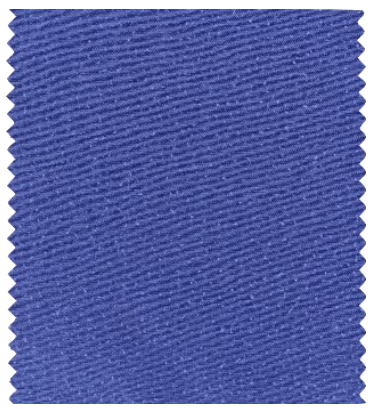

$85 \%$ Cotton

$10 \%$ Polyester

$5 \%$ Elastane

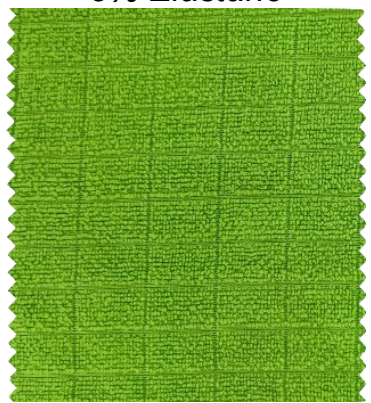

85\% Polyester

$15 \%$ Polyamide

Fig. 2 Samples of the fabrics used

A microscopic analysis of the textile fabrics was performed. The experimental setup shown in Figure 3 was used for this purpose. It consisted of a personal computer and a microscope. The microscope is Bresser BioLux NV, with serial number 61-15200. A 4x zoom lens is used. The microscope has two light sources - one for transmitted light and the second for reflection. Both sources use a white LED with the highest light intensity at $450 \mathrm{~nm}$. In the present work, the light source illuminating the sample from above is used.

Digital images were obtained with a MikrOkula eyepiece camera, with serial number 5913650 . It uses a CMOS color sensor. The size of the pixel is $3 \mu \mathrm{m} \times 3 \mu \mathrm{m}$, the size of the sensor is $5.86 \mathrm{~mm} \times 3.28 \mathrm{~mm}$. The camera has a standard diameter of $23.2 \mathrm{~mm}$ and is placed in place of the microscope eyepiece. It has an integrated UV/IR filter. The digital images of the used fabrics have a resolution of $640 \times 480$ pixels. 


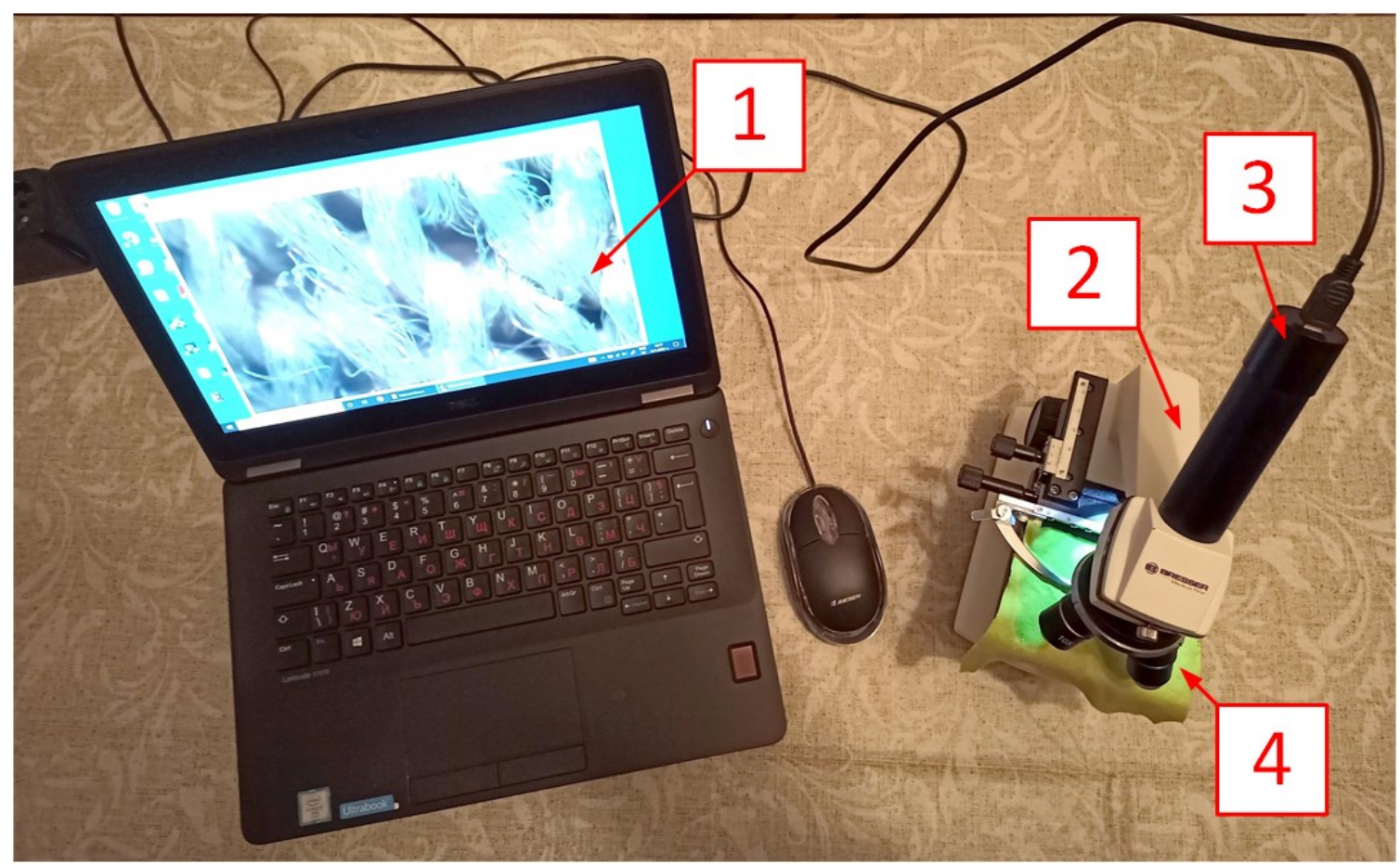

Fig. 3 Experimental set-up for obtaining microscopic images - general view. 1: personal computer with software; 2: microscope; 3: video camera; 4: measured sample

Figure 4 shows microscopic images of samples from different textile fabrics. They are used in the identification of fibers in textile fabrics.

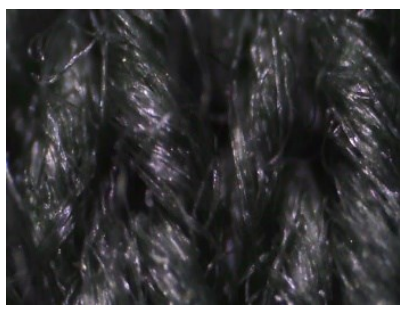

$100 \%$ Cotton

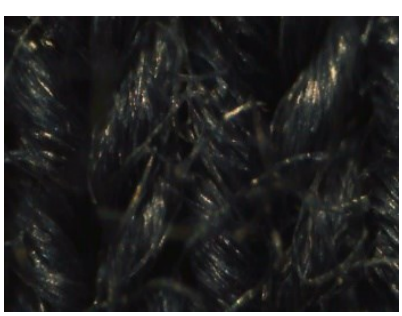

95\% Cotton

$5 \%$ Elastane

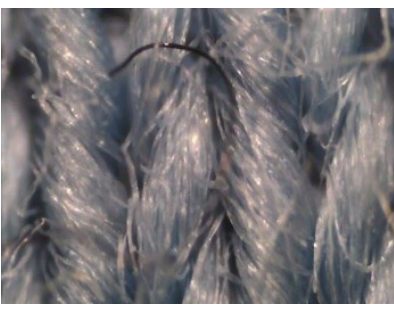

$100 \%$ Cotton

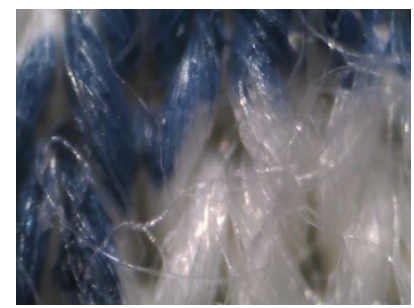

$85 \%$ Cotton

$15 \%$ Polyester

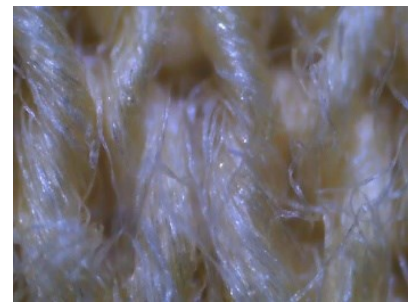

$100 \%$ Cotton

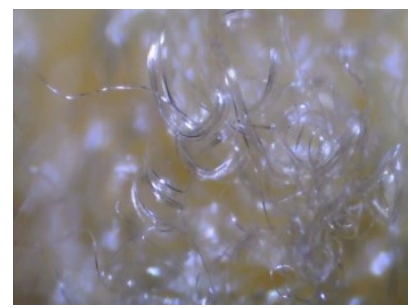

$85 \%$ Polyester $15 \%$ Polyamide

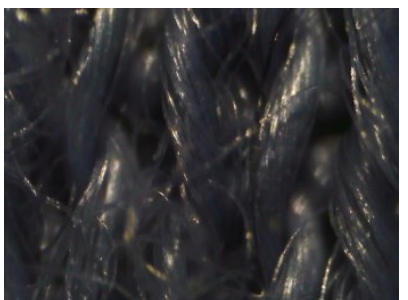

$85 \%$ Cotton

$10 \%$ Polyester

5\% Elastane

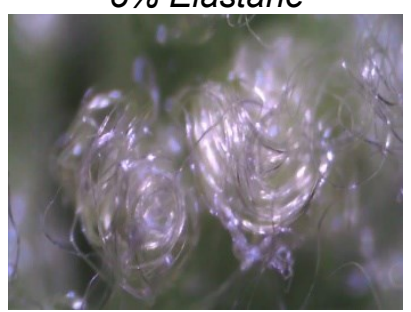

$85 \%$ Polyester $15 \%$ Polyamide

Fig. 4. Microscopic images of the samples used

The spectral characteristics of the textile fabrics were obtained by converting the values from the LMS color space model to the spectral characteristics in the VIS region. They are in the range 390-780 $\mathrm{nm}$. They are obtained by mathematical dependencies presented in the available literature [7]. The values of the color components of the RGB color model are converted to LMS. Using the matching functions, the spectral characteristics of the three channels $L, M$ and $S$ were calculated. After mathematical transformation, the spectral characteristics representing the reflection value for each wavelength in the above spectral range were calculated. 
Figure 5 shows the spectral characteristics of samples from different textile samples. In the three variants of $100 \%$ cotton samples, no visible difference in the obtained spectral characteristics was observed. Similarly, for $85 / 15 \%$ polyester/polyamide, there is no visible difference in the spectral characteristics obtained. Further analyzes are needed to establish the similarity or difference of the spectral characteristics and the features that can be derived from them.

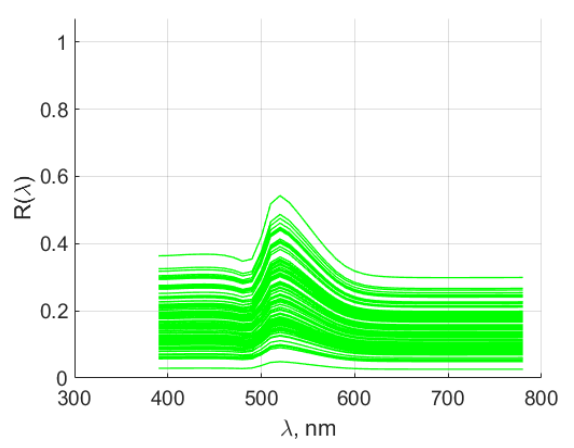

$100 \%$ Cotton

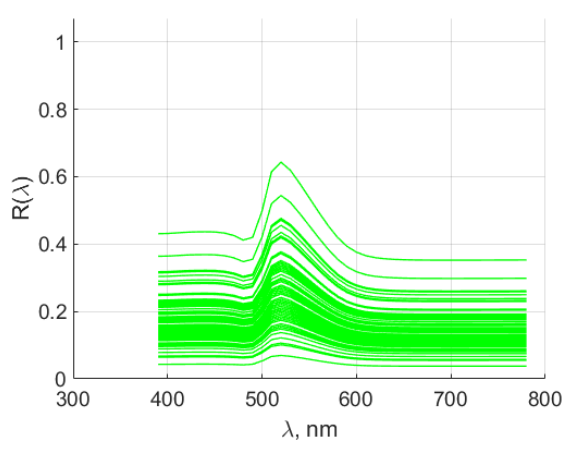

$85 \%$ Cotton $10 \%$ Polyester

$5 \%$ Elastane

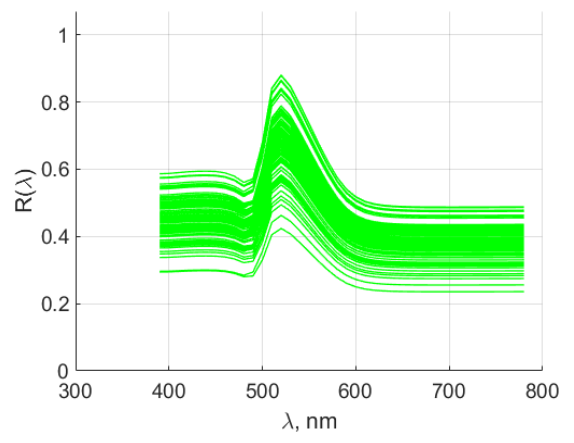

85\% Polyester $15 \%$ Polyamide

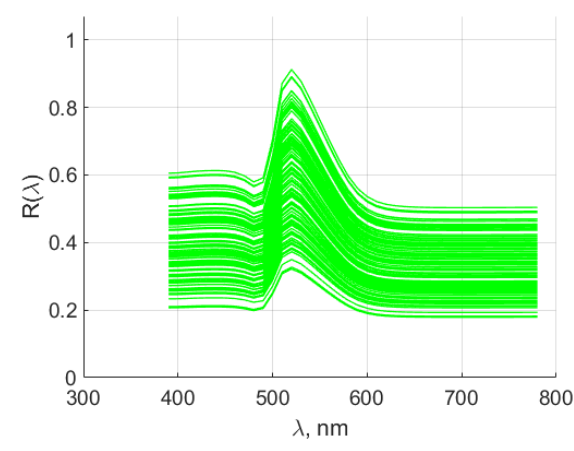

$100 \%$ Cotton

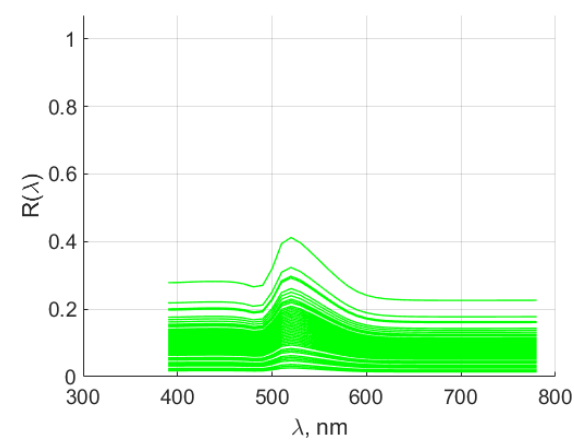

95\% Cotton 5\% Elastane

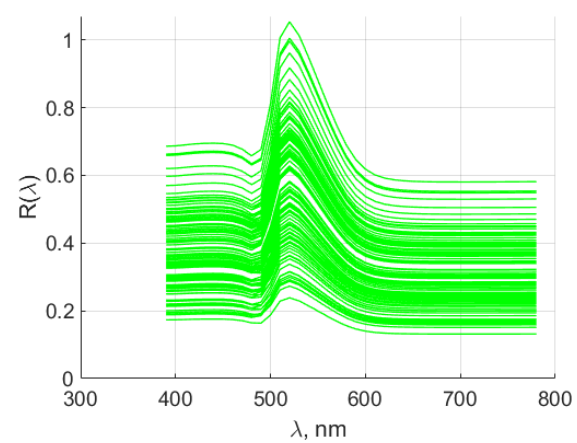

85\% Polyester $15 \%$ Polyamide

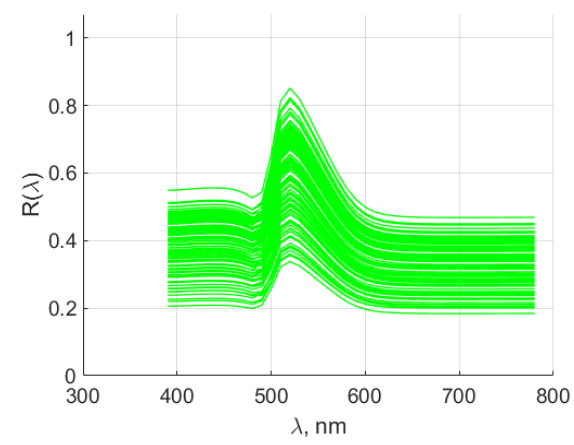

$100 \%$ Cotton

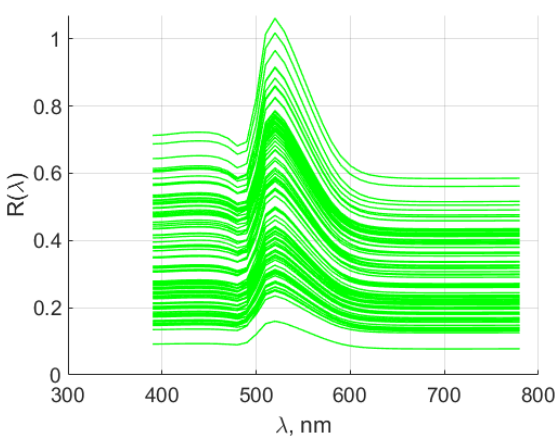

$85 \%$ Cotton $15 \%$ Polyester

Fig. 5. Spectral characteristics of the fabrics used

Spectral features were used according to Cermakova et al. [8] and Atanassova et al. [9]. The indicated wavelengths in the mathematical dependences are on the spectral characteristics of the reflection, in the visible range of the spectrum. They refer to the blue, green and red colors of the spectrum. The following formulas were used to calculate a total of 11 spectral features: 
$F_{1}=\frac{R_{740}}{R_{720}}$

$F_{2}=\frac{R_{530}-R_{570}}{R_{530}+R_{570}}$

$F_{3}=\frac{1}{R_{510}}-\frac{1}{R_{550}}$

$F_{4}=0.5\left(120\left(R_{750}-R_{550}\right)-200\left(R_{670}-R_{550}\right)\right)$

$F_{5}=\frac{R_{550}}{R_{680}}$

$F_{6}=\frac{2 R_{520}-R_{620}-R_{420}}{R_{520}+R_{620}+R_{420}}$

The influence of color on the spectral characteristics of fabrics with the same textile fibers, but with different color, was checked. Two hypotheses are accepted: $\mathrm{H}_{0}$ - there is no statistically significant difference between the groups; $\mathrm{H}_{1}$ - there is a statistically significant difference between the groups. A level of significance $\alpha=0.05$ and degrees of freedom (DF) were used. The $p$-value criterion was evaluated. If $p<\alpha$, the null hypothesis is rejected.

For the selection of informative features describing the spectral characteristics of textile fabrics, methods of successively improving evaluations have been used [10]. These methods reduce the number of feature combinations obtained. They are suitable for the selection of features for the classification of fabrics depending on the textile fibers used in them. There are three selection methods used:

- ReliefF - The method is a feature selection function using the ReliefF algorithm. It is suitable for choosing the features for classification models based on distance;

- FSNCA - In this method, the weight coefficients of the features are determined by adopting the method of analysis of neighborhood components (NCA). It uses distance-based functions;

- SFCPP - Through this method there is an optimal set of features that are mutually and maximally different and can effectively represent the compared objects. The algorithm minimizes the set of features by selecting the appropriate set of features describing the classified objects.

The reduction of the volume of data in the feature vectors was done by principal components (PC), obtained by the principal components analysis method. The other method used is latent variables (LV) obtained by the partial least squares regression method.

The following methods were used for classification [11]: Naïve Bayesian classifier (NB) - used as a reference; k-nearest neighbors (kNN); discriminant analysis (DA). Linear (L) and quadratic (Q) separation functions were used in the discriminant analysis.

After assessing the separability of the data with a naïve Bayesian classifier, these methods are selected, by which the lowest value of errors is obtained.

To evaluate the performance of the classifiers used, basic $\left(e_{i}\right)$, actual $\left(g_{i}\right)$ and total $\left(e_{0}\right)$ classification errors were calculated. The main error is an indicator of how much of the Class 1 data falls incorrectly into the other classes. The actual error shows the relative share of data from other classes incorrectly assigned by the classifier to Class 1 . The total error shows the incorrectly classified data relative to all data in the sample. 


\section{Results and discussion}

The results of the preliminary analyzes of spectral characteristics of fabrics made of $100 \%$ cotton, but with different color show that at $\mathrm{DF}=234-258$ and $p=0.00-0.03$ no statistically significant difference was observed between the compared groups. This shows that fabrics made of $100 \%$ cotton can be used as a general sample, regardless of their color.

Similar results were observed for the spectral characteristics of fabrics with $85 \%$ polyester and $15 \%$ polyamide. At $D F=196-199$ and $p=0.00-0.04$ no statistically significant difference was observed between the compared groups. Fabrics with these fibers are also considered as a general sample, regardless of their color.

A total of 5 groups of textile fabrics are formed, with the respective fibers from which they are made. These groups are shown in Table 1.

Table 1. Data on the samples used

\begin{tabular}{lll}
\hline Sample & Textile fiber composition & Number of spectral characteristics \\
\hline S1 & $100 \%$ Cotton & 100 \\
\hline S2 & $85 \%$ Cotton 10\% Polyester 5\% Elastan & 100 \\
\hline S3 & $95 \%$ Cotton 5\% Elastan & 100 \\
\hline S4 & $85 \%$ Cotton 15\% Polyester & 100 \\
\hline S5 & 85\% Polyester 15\% Polyamide & 100 \\
\hline
\end{tabular}

Figure 6 shows the results of the selection of informative features obtained from the spectral characteristics of the samples. The weight coefficients of the features obtained from the spectral characteristics are shown. Those features that have weight coefficients with a value above 0.6 are selected.

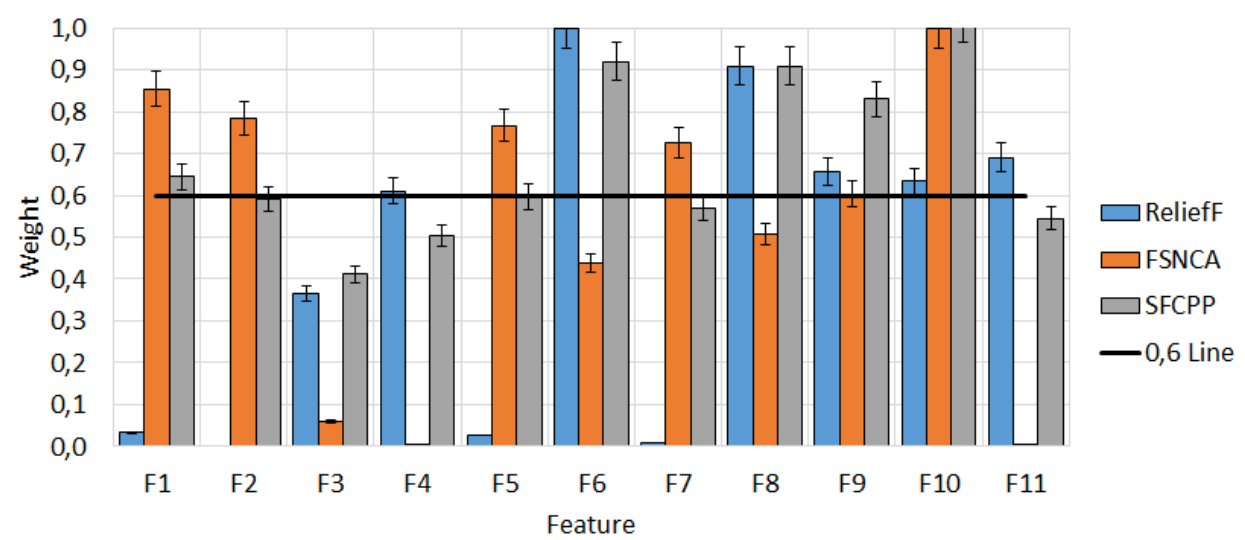

Fig. 6 Selection of informative features. F1-F11 denote features (spectral indices) described in equations (1)-(11)

The selected feature vectors are presented in Table 2. The feature vector FV1 consists of a total of 6 features. FV2 has 5 features. Most features are selected in FV3 - a total of 8 of them.

Table 2. Selected feature vectors

\begin{tabular}{lll}
\hline Feature vector & Method of selection & Selected features \\
\hline FV1 & ReliefF & F4, F6, F8, F9, F10, F11 \\
\hline FV2 & FSNCA & F1, F2, F5, F7, F10 \\
\hline FV3 & SFCPP & F1, F2, F5, F6, F8, F9, F10, F11 \\
\hline
\end{tabular}

The required number of principal components $(P C)$ and latent variables (LV) to which the data in the feature vectors can be reduced are determined. Figure 7 shows the result of this analysis. Two principal components and two latent variables are sufficient to describe more than $95 \%$ of the variance in the 
data. The experimental data from the feature vectors used for classification have been reduced to such an extent.

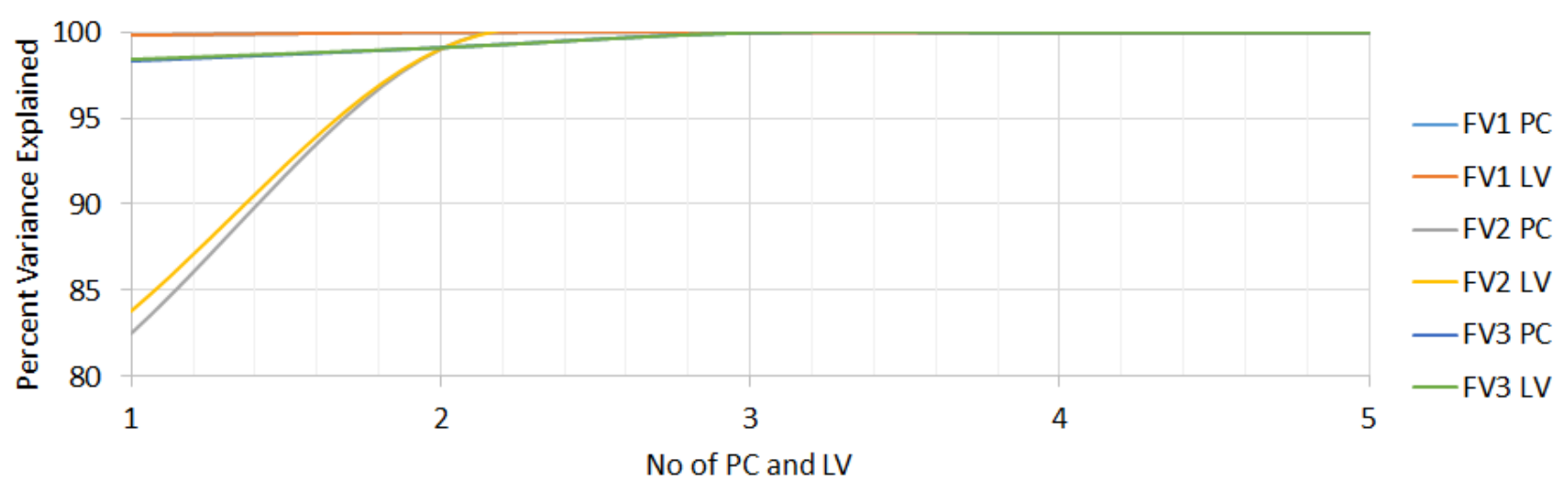

Fig. 7 Determining the required number of principal components and latent variables

Table 3 shows the results of a classification with a naive Bayesian classifier. When using FV1, reduced with both PC and LV, it is seen that the actual error has the largest values compared to the main and total error. This is an indicator that some of the data from the second class fall into the first. When reducing $\mathrm{FV} 1$ with $\mathrm{PC}$, the error values are smaller than those of $\mathrm{LV}$.

When using FV2 reduced with $\mathrm{PC}$, errors in the range of $0-3 \%$ are observed, these errors have the lowest values compared to other vectors of features and methods for reducing the volume of their data. In this feature vector, the use of LV again to reduce the volume of its data leads to significantly higher error values (above 10\%).

A similar trend is observed in data reduction and classification with $\mathrm{FV} 3$. When using a PC, the errors are in the range of $0-12 \%$, while when using an LV they are well over $10 \%$. Higher values of the actual error are observed, which shows that part of the data of the second class falls into the first.

Table 3. Results for a naïve Bayesian classifier

\begin{tabular}{|c|c|c|c|c|c|c|c|c|c|c|c|c|c|c|c|c|c|c|}
\hline \multirow{3}{*}{$\begin{array}{l}\text { FV } \\
\text { DRM } \\
\text { Error }\end{array}$} & \multicolumn{6}{|l|}{ FV1 } & \multicolumn{6}{|c|}{ FV2 } & \multicolumn{6}{|c|}{ FV3 } \\
\hline & \multicolumn{3}{|l|}{$\mathrm{PC}$} & \multicolumn{3}{|l|}{ LV } & \multicolumn{3}{|c|}{ PC } & \multicolumn{3}{|l|}{ LV } & \multicolumn{3}{|c|}{ PC } & \multicolumn{3}{|l|}{ LV } \\
\hline & $\mathrm{e}_{\mathrm{i}}$ & $g_{i}$ & $e_{0}$ & $e_{i}$ & $g_{i}$ & $\mathrm{e}_{0}$ & $\mathrm{e}_{\mathrm{i}}$ & $g_{i}$ & $\mathrm{e}_{0}$ & $e_{i}$ & $g_{i}$ & & & $g_{i}$ & $\mathrm{e}_{0}$ & & $g_{i}$ & \\
\hline S1-S2 & $3 \%$ & $6 \%$ & $5 \%$ & & $50 \%$ & & $1 \%$ & $0 \%$ & $0 \%$ & & $38 \%$ & & $2 \%$ & $0 \%$ & & $24 \%$ & $50 \%$ & \\
\hline -S & & & & & & & $0 \%$ & $\%$ & & & & & $\%$ & & & & $\%$ & \\
\hline S1-S4 & $6 \%$ & & 41 & $51 \%$ & & & $1 \%$ & $1 \%$ & & $58 \%$ & & & $4 \%$ & $8 \%$ & & $4 \%$ & $46 \%$ & \\
\hline S1-S5 & $3 \%$ & $49 \%$ & $65 \%$ & $47 \%$ & $1 \%$ & $63^{\circ}$ & $0 \%$ & $2 \%$ & 19 & $42 \%$ & $45 \%$ & & $0 \%$ & $12 \%$ & & $6 \%$ & $48 \%$ & $68 \%$ \\
\hline S2-S3 & $\%$ & $0 \%$ & $0 \%$ & $9 \%$ & $31 \%$ & $27 \%$ & $0 \%$ & $0 \%$ & $0 \%$ & $\%$ & $0 \%$ & $0 \%$ & $0 \%$ & $0 \%$ & $0 \%$ & $\%$ & $12 \%$ & $7 \%$ \\
\hline S2-S4 & $16 \%$ & $42 \%$ & $41 \%$ & $51 \%$ & $51 \%$ & $65 \%$ & $1 \%$ & $1 \%$ & $3 \%$ & $58 \%$ & $58 \%$ & $61 \%$ & $4 \%$ & $8 \%$ & $6 \%$ & $54 \%$ & $46 \%$ & $66 \%$ \\
\hline S2-S5 & $23 \%$ & $49 \%$ & $68 \%$ & $47 \%$ & $1 \%$ & $64 \%$ & $0 \%$ & $2 \%$ & $1 \%$ & $42 \%$ & $45 \%$ & $64 \%$ & $0 \%$ & $12 \%$ & & $6 \%$ & $48 \%$ & $68 \%$ \\
\hline & $5 \%$ & $42 \%$ & & 5 & & 59 & $1 \%$ & $1 \%$ & 20 & 58 & & & $4 \%$ & $8 \%$ & & $54 \%$ & $46 \%$ & \\
\hline & $3 \%$ & 94 & & $47 \%$ & 5 & 63 & $0 \%$ & $2 \%$ & & $42 \%$ & & & $0 \%$ & & & $36 \%$ & $48 \%$ & \\
\hline $4-S 5$ & $23 \%$ & $49 \%$ & $66 \%$ & $47 \%$ & $51 \%$ & $63 \%$ & $0 \%$ & $2 \%$ & $1 \%$ & $42 \%$ & $45 \%$ & $65 \%$ & $0 \%$ & $12 \%$ & $6 \%$ & $36 \%$ & $48 \%$ & $66 \%$ \\
\hline
\end{tabular}

FV-feature vector; DRM-data reduction method; PC-principal components; LV-Latent variables; basic (ei), actual $\left(g_{i}\right)$ and total $\left(\mathrm{e}_{0}\right)$ classification errors

Figure 8 shows the average error values using a naïve Bayesian classifier. The graph is a summary of the dependencies described above. The lowest error values were obtained by reducing the data volume of the feature vectors FV2 and FV3, with principal components (PC). In other cases, the errors significantly exceed $10 \%$. For the following analyses, a feature vector FV2, reduced by the principal components (PC) method, was chosen because the use of these data resulted in errors of less than $5 \%$ for all cases of textile fiber classification. 


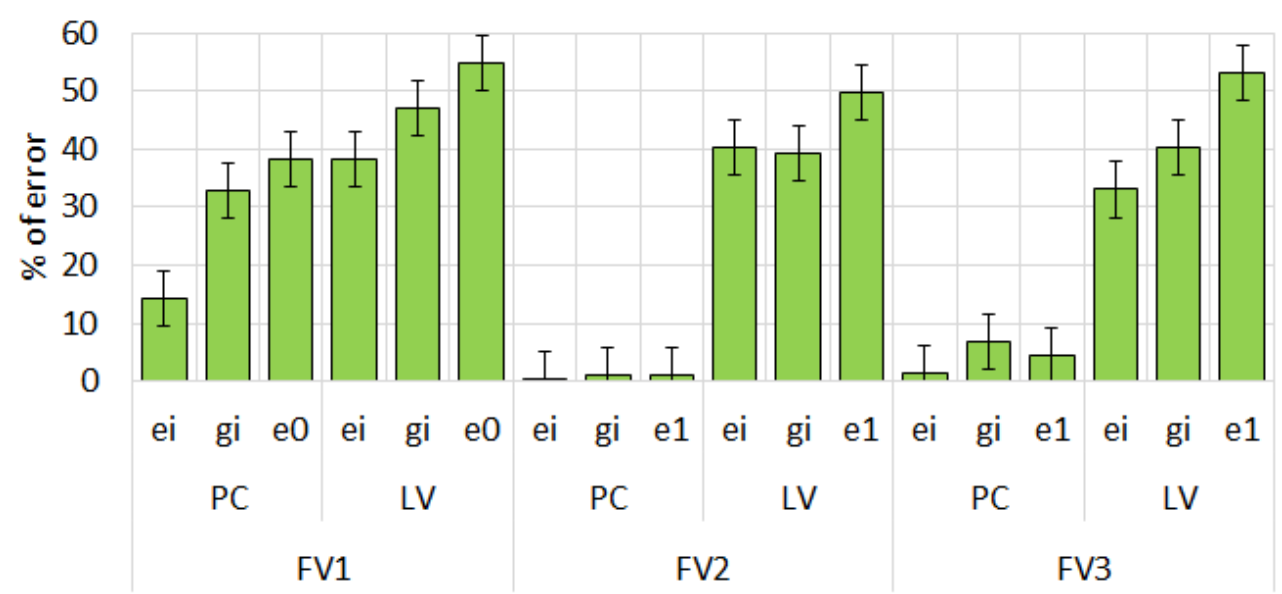

Error, DRM, FV

Fig. 8 Average values of errors in a naïve Bayesian classifier. DRM-data reduction method; FV-feature vector

Table 4 shows the results of the classification by FV2 data reduced by PC. When using the kNN method, significantly higher values of the main classification error are observed. This shows that the data from the first class fall incorrectly into the second one. Between classes S3-S4 there is an increase in the actual error, which indicates that class 1 includes data from the second class. Although the total classification error is $0 \%$, the use of $\mathrm{kNN}$ for the classification of textile fibers is inappropriate because more than $10 \%$ of the data are misclassified and do not fall into the relevant class to which they belong.

When using DA, regardless of the type of the separation function, uniform classification errors of up to $10 \%$ are obtained.

The obtained results show that the accuracy of classification depends on the features used in the vector, the method for reducing the volume of data, as well as the type of classifier. The accuracy of the classification does not depend on the type of the separation function.

Table 4. Results of kNN and DA

\begin{tabular}{l|lll|lll|lll}
\hline Classifier & $\mathrm{kNN}$ & \multicolumn{3}{l|}{} & \multicolumn{3}{|l|}{ DA-linear } & \multicolumn{3}{|l}{ DA-quadratic } \\
\hline Error & $\mathrm{e}_{\mathrm{i}}$ & $\mathrm{g}_{\mathrm{i}}$ & $\mathrm{e}_{0}$ & $\mathrm{e}_{\mathrm{i}}$ & $\mathrm{g}_{\mathrm{i}}$ & $\mathrm{e}_{0}$ & $\mathrm{e}_{\mathrm{i}}$ & $\mathrm{g}_{\mathrm{i}}$ & $\mathrm{e}_{0}$ \\
\hline S1-S2 & $25 \%$ & $0 \%$ & $0 \%$ & $0 \%$ & $0 \%$ & $9 \%$ & $0 \%$ & $0 \%$ & $9 \%$ \\
\hline S1-S3 & $31 \%$ & $0 \%$ & $0 \%$ & $0 \%$ & $0 \%$ & $9 \%$ & $0 \%$ & $0 \%$ & $9 \%$ \\
\hline S1-S4 & $43 \%$ & $0 \%$ & $0 \%$ & $0 \%$ & $0 \%$ & $9 \%$ & $0 \%$ & $0 \%$ & $9 \%$ \\
\hline S1-S5 & $35 \%$ & $0 \%$ & $0 \%$ & $0 \%$ & $0 \%$ & $9 \%$ & $0 \%$ & $0 \%$ & $9 \%$ \\
\hline S2-S3 & $31 \%$ & $0 \%$ & $0 \%$ & $0 \%$ & $0 \%$ & $9 \%$ & $0 \%$ & $0 \%$ & $9 \%$ \\
\hline S2-S4 & $35 \%$ & $0 \%$ & $0 \%$ & $0 \%$ & $0 \%$ & $9 \%$ & $0 \%$ & $0 \%$ & $9 \%$ \\
\hline S2-S5 & $35 \%$ & $0 \%$ & $0 \%$ & $0 \%$ & $0 \%$ & $9 \%$ & $0 \%$ & $0 \%$ & $9 \%$ \\
\hline S3-S4 & $57 \%$ & $36 \%$ & $0 \%$ & $0 \%$ & $0 \%$ & $9 \%$ & $0 \%$ & $0 \%$ & $9 \%$ \\
\hline S3-S5 & $35 \%$ & $0 \%$ & $0 \%$ & $0 \%$ & $0 \%$ & $9 \%$ & $0 \%$ & $0 \%$ & $9 \%$ \\
\hline S4-S5 & $24 \%$ & $0 \%$ & $0 \%$ & $0 \%$ & $0 \%$ & $9 \%$ & $0 \%$ & $0 \%$ & $9 \%$ \\
\hline
\end{tabular}

The results obtained in the present work complement those of the available literature.

Zhao et al. [6] claimed that portable spectrophotometric devices have the potential for application in the rapid and non-destructive differentiation of natural fibers in widely used fabrics. In the present work, spectral characteristics in the visible region of the spectrum are applied. This increases the potential for mass use of spectrophotometric techniques in the sorting of textile materials. 
The methods and procedures proposed here do not require high cost equipment and complex calculation procedures. They can be implemented in portable devices and microprocessor-based recognition systems. This complements research by Bergfjord et al. [2] in this area.

The question of the recognition of textile fibers when they are dyed with a dense and dark-colored dye remains open. The application of spectrophotometric techniques, regardless of the spectral range, is not sufficiently effective, as shown by Cura et al. [3]. One solution to this problem would be the joint use of spectrophotometric and other types of sensors such as inductive, capacitive, ultrasonic.

In the present work, a solution to the problem defined by Spahiu et al. and Tripa et al. [12-14] is suggested. The authors pointed out that there is potential growth in the market for products derived from the recovery, recycling and reuse of textiles, which necessitates the search for new solutions in this area.

In textile fabric recognition applications, the spectrometer and image acquisition and analysis systems are more often combined measuring instruments. In this way a better performance of the tasks of extracting information from the images of textile fabrics is achieved. Further work remains to be done on further developments related to the application of advanced optical methods, such as hyperspectral analysis. As a nondestructive method to distinguish fiber types, another important application of this method is for studies of cultural heritage collections and science conservation.

In our work, a comparative analysis of the effectiveness of algorithms for textile fabric classification was performed in terms of their classification error. Additional research is needed in the operation of the selected algorithms in their operation on single-board microcomputers, for example, Arduino or Raspberry Pi. Such an analysis would show the working speed of these algorithms and the possibilities for their direct implementation in real recognition systems, applicable directly to sorting lines for textile fabrics.

This work demonstrates the possibility of analyzing textile fabrics according to their visual images and spectral characteristics. Further analysis is needed for the effective use of the proposed tools. It must include the application of standard test methods described in ASTM D629-15 or ISO / TC 38 / SC 23 for fibers and yarns.

\section{Conclusion}

In the present work, an analysis of known methods and approaches applied so far using the techniques of spectral analysis is described. The proposed methods and procedures lead to improvement and facilitation of the process of classification of textile fibers in sorting and recycling of textile fabrics, in order to implement them in automated systems. The proposed analysis tools do not require high cost equipment and complex calculation procedures. They can be implemented in portable devices and microprocessor-based recognition systems.

It has been found that two principal components and two latent variables are sufficient to describe more than $95 \%$ of the variance in the data. This significantly reduces the amount of data used to analyze textile fibers by their spectral characteristics. It has been shown that the accuracy of classification of textile fibers does not depend on the type of separation function of the classifier used. This accuracy depends on the spectral characteristics used, the method for reducing the volume of data, and the type of classifier.

The obtained results can be used in the development of recognition systems for sorting textile fabrics depending on the composition of their fibers. In this way, the principles of sustainable fashion will be effectively applied. Also, the proposed methods and tools can be used in the training of future specialists in the subject area. 


\section{Acknowledgement}

The authors would like to express appreciation for the co-funding of the Erasmus+ Programme of the European Union [Project Fashion DIET "Sustainable fashion curriculum at textile Universities in Europe Development, Implementation and Evaluation of a Teaching Module for Educators" / Erasmus+ Programme 2020-1-DE01-KA203-005657] by the NA DAAD.

\section{References}

[1] Sabine Högsdal, Anne-Marie Grundmeier. 2021. Integrating design thinking in teacher education: student teachers develop learning scenarios for elementary schools. The International Journal of Design Education 16, 1, 1-26. DOI: https://doi.org/10.18848/2325-128X/CGP/v16i01/1-26.

[2] Christian Bergfjord, Bodil Holst. 2010. A procedure for identifying textile bast fibres using microscopy: Flax, nettle/ramie, hemp and jute. Ultramicroscopy 110, 9, 1192-1197. DOI: https://doi.org/10.1016/j.ultramic.2010.04.014.

[3] Kirsti Cura, Niko Rintala, Taina Kamppuri, Eetta Saarimäki, Pirjo Heikkilä. 2021. Textile recognition and sorting for recycling at an automated line using near infrared spectroscopy. Recycling 6, 1, 11. DOI: https://doi.org/10.3390/recycling6010011.

[4] Stanimir Karapetkov, Lubomir Dimitrov, Hristo Uzunov, Silvia Dechkova. 2019. Identifying vehicle and collision impact by applying the principle of conservation of mechanical energy. Transport and Telecommunication 20, 3, 191-204. DOI: https://doi.org/10.2478/ttj-2019-0016.

[5] J. Liu, L. Zou, F. Lv, Q. An, J. Liu, H. Men. 2015. Application of Spectroscopy Technology in Textiles. Proceedings of International Conference of Electrical, Automation and Mechanical Engineering (EAME 2015), 482-484. DOI: https://doi.org/10.2991/EAME-15.2015.135.

[6] Hengqian Zhao, Yunli Wang, Shuai Liu, Kunheng Li, Wei Gao. 2019. Spectral reflectance characterization and fiber type discrimination for common natural textile materials using a portable spectroradiometer. Journal of Archaeological Science 111, 105026. DOI: https://doi.org/10.1016/j.jas.2019.105026.

[7] Chris Wyman, Peter-Pike Sloan, Peter Shirley. 2013. Simple Analytic Approximations to the CIE XYZ Color Matching Functions. Journal of Computer Graphics Techniques JCGT 2, 2, 1-11.

[8] Ivana Čermáková, Jitka Komárková, Pavel Sedlak. 2019. Calculation of visible spectral indices from UAVbased data: small water bodies monitoring. In 2019 14th Iberian Conference on Information Systems and Technologies CISTI, 1-5.

[9] Stefka Atanassova, Petar Nikolov, Nikolay Valchev, Stoyka Masheva, Dimitar Yorgov. 2019. Early detection of powdery mildew (Podosphaera xanthii) on cucumber leaves based on visible and near-infrared spectroscopy. AIP Conference Proceedings 2075, 160014.

[10] Plamen Daskalov, Eleonora Kirilova, Violeta Mancheva, Tsvetelina Georgieva. 2019. Comparative study on the application of visible and near infrared hyperspectral imaging for Fusarium disease assessment of corn seeds. WSEAS Transactions on Electronics 10, 72-80.

[11] Mirolyub Mladenov, Stanislav Penchev, Martin Deyanov. 2015. Complex assessment of food products quality using analysis of visual images, spectrophotometric and hyperspectral characteristics. International Journal of Engineering and Innovative Technology (IJEIT) 4, 12, 23-32.

[12] Tatjana Spahiu, Eriseta Canaj, Ermira Shehi. 2020. 3D printing for clothing production. Journal of Engineered Fibers and Fabrics 15, 1558925020948216. DOI: https://doi.org/10.1177/1558925020948216.

[13] T. Spahiu, A. Manavis, Z. Kazlacheva, H. Almeida, P. Kyratsis. 2021. Industry 4.0 for fashion products - Case studies using 3D technology. IOP Conference Series: Materials Science and Engineering 1031, 1, 012039. DOI: https://doi.org/10.1088/1757-899X/1031/1/012039.

[14] Simona Tripa, Liliana Indrie. 2021. Households textile waste management in the context of a circular economy in Romania. Environmental Engineering and Management Journal 20, 1, 81-87. 